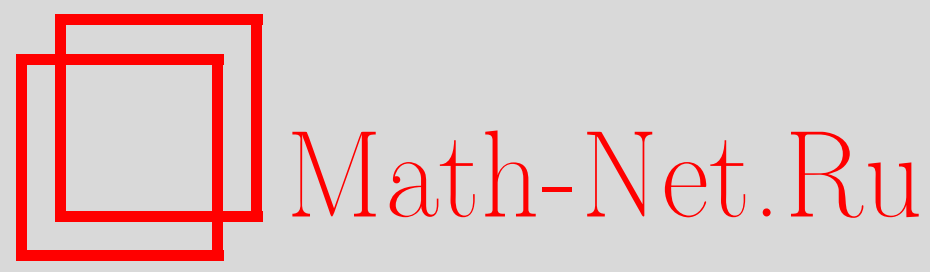

А. И. Штерн, Деформация неприводимых унитарных представлений дискретной серии эрмитово симметрических простых групп Ли в классе чистых псевдопредставлений, $M a$ тем. заметки, 2003, том 73, выпуск 3, 478-480

DOI: https://doi.org/10.4213/mzm622

Использование Общероссийского математического портала Math-Net.Ru подразумевает, что вы прочитали и согласны с пользовательским соглашением http://www . mathnet.ru/rus/agreement

Параметры загрузки:

IP: 54.237 .206 .68

26 апреля 2023 г., 14:03:10

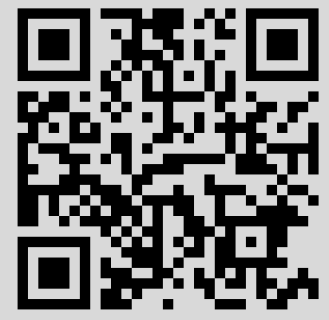




\section{ДЕФОРМАЦИЯ НЕПРИВОДИМЫХ УНИТАРНЫХ ПРЕДСТАВЛЕНИЙ ДИСКРЕТНОЙ СЕРИИ ЭРМИТОВО СИММЕТРИЧЕСКИХ ПРОСТЫХ ГРУПП ЛИ В КЛАССЕ ЧИСТЫХ ПСЕВДОПРЕДСТАВЛЕНИЙ}

\section{А. И. Штерн}

1. Формулировка основного результата. Напомним, что простая группа Ли называется эрмитово симметрической, если центр ее универсальнй накрывающей группы бесконечен (см. [1]). Кроме того, напомним, что для данного $\varepsilon \geqslant 0$ отображение $\pi: G \rightarrow L(H)$ группы $G$ в пространство линейных операторов в гильбертовом пространстве $H$ называется унитар-

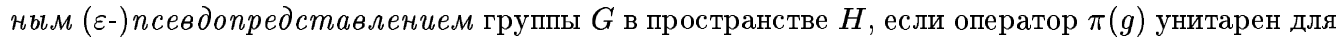
любого $g \in G$ и $\|\pi(g h)-\pi(g) \pi(h)\| \leqslant \varepsilon$ для любых $g, h \in G[2]$. Псевдопредставление $\pi$ называется чистым, если ограничение $\pi$ на любую аменабельную подгруппу $H$ группы $G$ является обычным унитарным представлением группы $H$. Это условие является весьма ограничительным, так как псевдопредставление может не удовлетворять этому условию даже на подгруппах, порожденных одним элементом (см. [3]).

Оказьвается, неприводимые унитарные представления простых эрмитово симметрических групп Ли (в том числе описанные в [4] неприводимые унитарные представления дискретной серии для таких групп Ли, которые не могут быть непрерьвно деформированы в классе унитарных представлений), могут быть непрерывно деформированы в классе чистых унитарных псевдопредставлений. А именно, имеет место следующее утверждение.

Теорема. Пусть $G$ - простая эрмитово симметрическая группа Ли. Для любого (сильно непрерывного) неприводимого унитарного представления $\sigma$ группь $G$ в некотором гильбертовом пространстве $H$ существует (сильно непрерывное по параметру) однопараметрическое семейство $t \mapsto \pi_{t}^{\sigma}, t \in \mathbb{R}$, попарно неэквивалентных чистых неприводимых унитарных $\varepsilon_{t}$-псевдопредставлений группы $G$ в том же пространстве $H$, удовлетворяющ,е следующим двум условиям:

1) $\varepsilon_{t} \rightarrow 0$ npu $t \rightarrow 0$

2) норма $\left\|\pi_{t}^{\sigma}(g)-\sigma(g)\right\|$ стремится $\kappa$ нулю при $t \rightarrow 0$ для любого $g \in G$.

В настоящей заметке выписаны явные формулы, относящиеся к модельному случаю $G=\mathrm{SL}(2, \mathbb{R})$ или, что эквивалентно, к случаю изоморфной группы $\mathrm{SU}(1,1)$. Полное доказательство теоремы будет приведено в одной из следующих публикаций.

2. Деформация неприводимых унитарных представлений группы $\mathrm{SU}(1,1)$. Примем обозначения, использованные в [5]. Пусть $\widetilde{G}-$ универсальная накрывающая группа группы $\mathrm{SU}(1,1)$. Мы воспользуемся ее стандартной параметризацией: $\widetilde{G}=\{(\gamma, \omega)|\gamma \in \mathbb{C},| \gamma \mid<1$, $\omega \in \mathbb{R}\} ;$ групповая операция имеет вид $(\gamma, \omega)\left(\gamma^{\prime}, \omega^{\prime}\right)=\left(\gamma^{\prime \prime}, \omega^{\prime \prime}\right),(\gamma, \omega),\left(\gamma^{\prime}, \omega^{\prime}\right),\left(\gamma^{\prime \prime}, \omega^{\prime \prime}\right) \in \widetilde{G}$, где

$$
\begin{aligned}
\gamma^{\prime \prime} & =\left(\gamma+\gamma^{\prime} \exp \left(-2 i \omega^{\prime}\right)\right)\left(1+\bar{\gamma} \gamma^{\prime} \exp (-2 i \omega)\right)^{-1}, \\
\omega^{\prime \prime} & =\omega+\omega^{\prime}+\frac{1}{2 i} \ln \left\{\left(1+\bar{\gamma} \gamma^{\prime} \exp (-2 i \omega)\right)\left(1+\gamma \bar{\gamma}^{\prime} \exp (2 i \omega)\right)^{-1}\right\} .
\end{aligned}
$$

Здесь $\ln$ понимается в смысле главного значения.

Пусть $\mathbb{T}$ - одномерньй тор. Определим действие группы $\widetilde{G}$ на $\mathbb{T}$ формулой

$$
\exp (i \theta) \cdot g=\exp (2 i \omega)\left(\frac{\exp (i \theta)+\gamma}{\exp (i \theta) \bar{\gamma}+1}\right)
$$

Работа выполнена при поддержке Российского фонда фундаментальных исследований, грант № 02-01-00574. 
Напомним определение представлений основной серии группы $\widetilde{G}[5,(2.2 .9)]$. Для любых $\rho \in \mathbb{R}$ и $h \in(-1 / 2,1 / 2]$ формула

$$
\begin{aligned}
& {\left[U_{h}(g, \rho) f\right](\exp (i \theta))} \\
& \quad=\exp (-2 i \omega h)\left(\frac{1+\exp (i \theta) \bar{\gamma}}{1+\exp (-i \theta) \gamma}\right)^{h}|\exp (i \theta) \bar{\beta}+\bar{\alpha}|^{-1-2 i \rho} f(\exp (i \theta) \cdot g), \\
& \quad f \in H=L^{2}(\mathbb{T})
\end{aligned}
$$

где

$$
\alpha=\exp i \omega\left(1-|\gamma|^{2}\right)-\frac{1}{2} \quad \text { и } \quad \beta=\exp i \omega \gamma\left(1-|\gamma|^{2}\right)-\frac{1}{2}
$$

являются матричными элементами образа $g_{0}$ элемента $g \in \widetilde{G}$ в $\mathrm{SU}(1,1)$, а именно:

$$
g_{0}=\left(\begin{array}{ll}
\alpha & \bar{\beta} \\
\beta & \bar{\alpha}
\end{array}\right)
$$

определяет неприводимое (за исключением случая $\rho=0$ и $h=1 / 2$ ) унитарное представление группы $\widetilde{G}$ в гильбертовом пространстве $H$.

Для построения искомой деформации неприводимьх унитарных представлений основной серии группы $\mathrm{SU}(1,1)$ мы используем нетривиальный псевдохарактер $\chi$ на группе $\widetilde{G}[6]$, определенный формулой

$$
\chi((\gamma, \omega))=\lim _{n \rightarrow \infty} n^{-1} \omega_{n}, \quad \text { где }(\gamma, \omega)^{n}=\left(\gamma_{n}, \omega_{n}\right) \in \widetilde{G} \text { при } n \in \mathbb{N} .
$$

Явное выражение для псевдохарактера $\chi$ вычислено в [7]. В частности, там показано, что функция $\chi$ удовлетворяет условиям

$$
\left|\chi\left((\gamma, \omega)\left(\gamma^{\prime}, \omega^{\prime}\right)\right)-\chi((\gamma, \omega))-\chi\left(\left(\gamma^{\prime}, \omega^{\prime}\right)\right)\right|<\frac{\pi}{2}
$$

для любых $(\gamma, \omega),\left(\gamma^{\prime}, \omega^{\prime}\right) \in \widetilde{G}$, причем ограничение функции $\chi$ на любую аменабельную подгруппу в $\widetilde{G}$ является непрерывным гомоморфизмом этой подгруппы в группу $\mathbb{R}$. По этой причине формула $\psi_{t}(g)=\exp (i t \chi(g)), g \in \widetilde{G}$, определяет функцию $\psi_{t}: \widetilde{G} \rightarrow \mathbb{T}$, удовлетворяющую условию

$$
\left|\psi_{t}\left(g g^{\prime}\right)-\psi_{t}(g) \psi_{t}\left(g^{\prime \prime}\right)\right| \leqslant \sup _{|\phi|<\pi / 2}|\exp (i t \phi)-1|, \quad g, g^{\prime} \in \widetilde{G} .
$$

При $|t|<2$ это приводит к неравенству

$$
\left|\psi_{t}\left(g g^{\prime}\right)-\psi_{t}(g) \psi_{t}\left(g^{\prime}\right)\right|<\left|\exp \left(\frac{i t \pi}{2}\right)-1\right|, \quad g, g^{\prime} \in \widetilde{G} .
$$

Отсюда следует, что при данном $\rho \in \mathbb{R}$ формула

$$
\pi_{h}^{\rho}(g)=\psi_{2 h}(g) U_{h}(g, \rho), \quad g \in \widetilde{G},
$$

определяет чистое псевдопредставление группы $\widetilde{G}$ унитарньми операторами в пространстве $L^{2}(\mathbb{T})$ для любого $h \in \mathbb{R}$. Неприводимость семейства $\pi_{h}^{\rho}(g), g \in \widetilde{G}$, следует из неприводимости представлений основной серии [5]. Центр $Z$ группы $\widetilde{G}$ образован элементами вида $(0, n \pi), n \in \mathbb{Z}$, и согласно (1) они переходят в единичный оператор: $\pi_{h}^{\rho}((0, n \pi))=1_{L^{2}(\mathbb{T})}$. Для любого $g \in \widetilde{G}$ подгруппа $A g$, порожденная $g$ и $Z$, аменабельна. Поэтому ограничение чистого псевдопредставления $\pi_{h}^{\rho}$ на эту подгруппу есть обычное унитарное представление группы $A_{g}$. Следовательно, $\pi_{h}^{\rho}(g(0, n \pi))=\pi_{h}^{\rho}(g)$ для любого $g \in \widetilde{G}$. Таким образом, $\pi_{h}^{\rho}$ постоянна на смежных классах по центру, и потому определяет чистое псевдопредставление факторгруппы группы $\widetilde{G}$ по ее центру и, в частности, чистое псевдопредставление группы $\mathrm{SU}(1,1)$. Ограничение каждого из построенных 
чистых псевдопредставлений группы $\mathrm{SU}(1,1)$ на максимальную компактную подгруппу диагональных матриц $K \subset \mathrm{SU}(1,1)$ содержит ненулевой $K$-инвариантный вектор, и соответствующие матричные элементы различны при различных $h \in \mathbb{R}$; следовательно, чистые псевдопредставления $\pi_{h}^{\rho}$ попарно неэквивалентны. Утверждения 1) и 2) теоремы проверяются непосредственно, и, тем самым, формула $h \mapsto \pi_{h}^{\rho}, h \in \mathbb{R}$, определяет искомую деформацию представлений основной серии $\pi^{\rho}$, отвечающих $h=0$.

Деформация неприводимых унитарных представлений второй части основной серии (отвечающих значению $h=1 / 2$ ) строится совершенно аналогичным образом. Кроме того, тем же приемом получается деформация представлений дополнительной серии. Наконец, аналогичные формулы $\pi_{t}^{ \pm, h}(g)=\psi_{t}(g) U^{ \pm}(g, h), t \in \mathbb{R}, h \in \mathbb{R}, \pm h>0, g \in \widetilde{G}$, определяют деформацию представлений дискретной серии группы $\mathrm{SU}(1,1)$, отвечающих знаку \pm и полуцелому числу $\pm \nu$, если $t=h \mp \nu \in \mathbb{R}$, а $\nu$ - положительное полуцелое число.

Автор благодарен А. С. Мищенко за внимание к работе и рецензенту за замечания, позволившие улучшить изложение.

\section{СПИСОК ЦИТИРОВАННОЙ ЛИТЕРАТУРЫ}

1. Хелгасон С. Дифференциальная геометрия и симметрические пространства. М.: Мир, 1964. 2. Штерн А. И. // Функцион. анализ и его прилож. 1991. Т. 25. № 2 . С. 70-73. 3. Shtern А. I. // Russian J. Math. Physics. 1994. V. 2. № 3. P. 353-382. 4. Schmid W. // Invent. Math. 1975. V. 30. №1. P. 47-144. 5. Sally P. J., Jr. Analytic Continuation of the Irreducible Unitary Representations of the Universal Covering Group of SL $(2, \mathbb{R})$. Mem. Amer. Math. Soc. V. 69. Providence (R.I.): Amer. Math. Soc., 1967. 6. Shtern A. I. // Russian J. Math. Physics. 2001. V. 8. № 1. P. 115-126. 7. Shtern A. I. // Ann. Global Anal. Geom. 2001. V. 20. № 3. P. 199-221. 